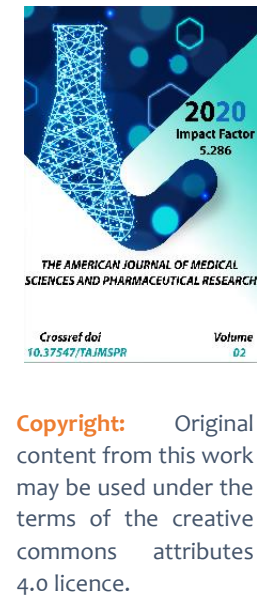

\section{Clinical And Functional Changes In Hard Tissues Of Teeth In Patients With Hemophilia}

\author{
Alisher Berdikulovich Norbutaev \\ Samarkand State Medical Institute, Uzbekistan \\ Mukhiddin Kamariddinovich Shamsiev \\ Samarkand State Medical Institute, Uzbekistan \\ Nodira Sharipovna Nazarova \\ Samarkand State Medical Institute, Uzbekistan
}

\title{
ABSTRACT
}

Hereditary coagulopathies characterized by reduced blood clotting occupy a special place among diseases of the blood system. The most common of these are hemophilia and Willebrand's disease. Hemophilia-the disease of "royals" - is an old, but still relevant disease.

Hereditary coagulopathies, characterized by reduced blood clotting, occupy a special place among diseases of the blood system. Based on who data and the likely frequency of research on these diseases, the prevalence of hemophilia ranges from 3 to 25 patients per 10,000 population.

Few studies of the oral cavity in patients with hemophilia have shown a high level of dental diseases, poor hygiene of the oral cavity. This is due to the lack of proper oral care, and dispensary monitoring, fear of prolonged bleeding after dental manipulations. For this reason, dentists try to avoid treating dental diseases in this category of patients.

At the same time, modern achievements of medical science in the field of Hematology and the development of effective replacement therapy allow to prevent almost all types of bleeding.

However, this group of patients is still unable to receive timely and high-quality dental treatment and prevention, which largely determines the high prevalence of inflammatory diseases of the maxillofacial region.

All of the above is evidence of the presence of many predisposing factors of damage to the dental system in patients with hemophilia, as well as the need for preventive, therapeutic, including orthopedic dental measures for this category of patients.

\section{KEYWORDS}

Hemophilia, hard and soft tissues of the oral cavity, Willebrand's disease, orthopedic treatment measures, dental status. 


\section{INTRODUCTION}

The main and almost the only sign of hemophilia is a tendency to bleed. People with a severe form of the disease often have spontaneous bleeding, and blood clotting tests always detect pathology. In the moderate form, spontaneous bleeding may occur after minor injuries, and the activated partial thromboplastin time (APTT) is always increased. The mild form is manifested by a tendency to bleeding after injuries and operations, spontaneous bleeding does not occur, blood clotting tests may be within the normal range. With a mild form of the disease, the diagnosis is usually made in adulthood.

\section{PURPOSE OF RESEARCH}

To study the clinical and functional changes of the oral organs in patients with hemophilia and to develop features of dental prosthetics.

\section{OBJECT AND SUBJECT OF RESEARCH}

We examined 30 patients with hemophilia at the research Institute of Hematology and blood transfusion of the Republic of Uzbekistan (Director Professor Karimov Kh.Ya.) aged 15 to 45 years. Practical healthy patients who applied to the dental clinic of the Department of orthopedic dentistry and orthodontics of TMA served as a control.

\section{RESULTS AND DISCUSSION}

We determined the need for dental care in patients with hemophilia, assessed the condition of the oral mucosa, bleeding gums, the condition of existing dentures, and also assessed the state of microcirculation of the oral mucosa.

Patients ' complaints differed depending on the specific therapy and the volume of dental care. When entering the clinic, patients complain more about bleeding gums, pain when eating. After oral sanitation and specific therapy, patients ' complaints increase, and tooth mobility appears against the background of increased bleeding.

If the bleeding was due to mechanical side effects of the dentures, it decreased after correction or removal of the dentures. Patients complained of hyperesthesia of the teeth and impaired taste sensitivity, a fetid smell from the mouth and lack of appetite. When examining the organs of the oral cavity, almost all patients have changes. Changes in the mucosa in the form of ulcerative, ulcerative-necrotic, hemorrhage, atrophy or hypertrophy, usually reflected the severity of the underlying disease.

Stomatoscopic examination of the gingival mucosa, lips and cheeks was performed in both the control group and the main group. When examining the mucosa of the alveolar ridge of almost healthy people, capillary networks are clearly visible against the background of pink color. Capillaries consist of an arterial and a venous part. The venous part is darker in color and much wider than the arterial part. The subcapillary part is not visible. Closer to the transition fold, the color of the mucosa is darker with a distinct pattern of the venous network, where the subcapillary network is clearly visible. Arterioles, precapillaries, capillaries, postcapillary venules, and major veins are clearly visible in this zone.

The surface blood supply is rich in straight arterioles, slightly sinuous capillaries, and moderately convoluted venules. Uniform blood flow is observed in both arterioles and venules. In the latter, aggregation of red blood cells with a fine-grained character is sometimes noted. 
The dental picture of the alveolar ridge mucosa in patients with hemophilia differs from the control one with a high degree of variability in both vascular and non-vascular changes.

In hemophilia, the mucosa of the alveolar ridge is pale, and there are often no signs of gingivitis. Correctly positioned capillaries have almost the same diameter, both in the arterial and venous parts. The capillaries are pink in color. The capillary network is clearly visible, both in the area of the transitional fold and in the area of the upper lip mucosa. The mucous membrane of the lower lip is darker in color with signs of stagnation.

On the surface of the gum mucosa, hyperplasia with bleeding phenomena is noted, the background color is pale with a bluish tinge, sometimes purple.

The capillaries are short and thickened. The subcapillary network is not visible. As pointed out above, there is stasis of blood in capillaries, edema and perivascularly many microhemorrhage.

Often gipertroficski gingivitis is accompanied by the muddy colour of the mucosa in the area of transitional fold. The capillary network is indistinct with pronounced recapillary edema, the subcapillary network is poorly visible, and a large number of hemorrhages.

Some patients have atrophied mucous membrane of the gums with a pale yellow tint. Capillaries are poorly visible, they are thin, short and sinuous.

The papillae between the teeth are swollen with dark blue, and sometimes purple color. There is a gray-yellow discharge from under the papillae with a fetid smell. On the surface of the papillae are capillaries, convoluted with signs of stasis. Spot hemorrhages are common.

On the surface of the mucous membrane of the transitional folds, the vascular network is clearly visible, but it is difficult to identify all the links of the microcirculatory bed. Observed perivascularly swelling. More, there are foci of hemorrhage. Minor mechanical damage contributed to the bleeding.

In patients who use removable prostheses, there is an imprint of the prosthesis base on the mucosa of the prosthetic bed, often in the area of transitional folds there is a non-healing ulcer that constantly bleeds.

As a rule, one patient had several complaints. Dry mouth was observed in 9 (30\%) patients. 27 (90\%) patients complained of bleeding gums, more often the bleeding was causal. In 30\% of cases, complaints were about pain, which was most often caused by the presence of pathological changes in the mucous membrane and periodontal tissues. Sometimes there were no changes in the mucosa, but patients complained of paresthesia, as well as a violation of taste sensitivity.

Ulcerative-necrotic lesions occurred in 3 (10\%) patients. Gum necrosis was more often observed in the area of bridges and crowns. Ulcers in the cheeks, tongue, and lips developed at the site of hemorrhages or as a result of mucosal trauma with the sharp edges of destroyed teeth. The sores were covered with a dirty gray coating, which then soaked in blood and took on a dark brown or even black color. Raids were hardly removed with tweezers and when they were removed, bleeding surfaces were exposed, sharply painful. 
An unpleasant smell emanated from the mouth. As a rule, there was no inflammatory reaction of the surrounding tissues. In some cases, mild hyperemia and mucosal edema were detected in the circumference of the ulcer.

Gum bleeding was mostly causal and manifested in all stages of the disease. The intensity of the hemorrhagic syndrome was different: from minor local hemorrhages to diffuse bleeding from the entire marginal surface of the gum.

Often, patients are disturbed not so much by heavy bleeding from the dental wells, but by the formation of blood clots attached to these wells that SAG into the oral cavity and prevent the patient from eating and talking. After colliding such clots with the tongue or finger, bleeding resumes with the re-formation of sagging clots. Bleeding gums were accompanied by hemorrhage in the oral mucosa of various locations: on the lips, cheeks, tongue, and palate.

Thus, in cases of hemophilia, there are significant clinical and stomatoscopic changes in the oral cavity, consisting in the presence of hypertrophy and edema of the gums, atrophy of the alveolar ridge tissue, and bleeding of the mucous membrane in all areas of the oral cavity. The revealed clinical features of the course of the disease cause differentiated approaches to diagnosis, determination of severity and treatment tactics in hemophilia.

Examination of the oral cavity in patients with hemophilia showed that all patients have dental caries. The prevalence of caries in all age groups was $100 \%$.

As a control, a dental study was conducted in 20 somatically healthy individuals, age groups corresponding to patients with hemophilia. Comparative analysis showed that the prevalence of caries in the control group ranged from $86.7 \%$ to $95.3 \%$. The average rate in somatically healthy individuals is $91.6 \%$.

Thus, with increasing age in the control group, the prevalence of dental caries increases, and in patients with hemophilia, it remained the same and was equal to $100 \%$.

The intensity of dental caries in patients depended on the severity of hemophilia. The analysis showed that the average number of carious teeth in patients with hemophilia was 6 times higher than in practically healthy individuals. The severity of hemophilia is reflected in the value of this indicator. The average number of sealed teeth in patients with hemophilia was significantly higher compared to the control group.

Regardless of the severity of hemophilia, the number of removed teeth was less than in the control ones. The intensity of dental caries depends on the age of the subjects and increases with increasing age both in the group of patients with hemophilia and in practically healthy individuals.

Thus, the examination of the oral cavity in 30 patients with hemophilia and 20 healthy individuals (control) showed that all patients were diagnosed with dental caries. With increasing age and severity of hemophilia, the intensity of caries significantly increased. In patients with hemophilia, the high and very high degree of caries activity was almost 2.5 times higher than the control indicators. 


\section{CONCLUSIONS}

All patients with hemophilia had lesions of the dentoalveolar system, the degree of which depended on the severity and duration of the underlying disease.

In $100 \%$ of cases of hemophilia, dental caries was observed, the intensity of which is associated with the severity of hemophilia. In patients with severe hemophilia, caries occurs 2 times higher than in the group of patients with a mild degree. With increasing age, the intensity of dental caries increased.

Patients with hemophilia have a low level of oral hygiene: the Fedorov-Volodkina index was 2.5 times higher than in somatically healthy individuals, and the UIGR gave an unsatisfactory assessment of oral hygiene in $90 \%$ of patients.

The prevalence of periodontal diseases in hemophilia was high and amounted to $82 \%$. Indicators of periodontal PMA indices exceeded the control values by 3 or more times, which indicates severe periodontal tissue damage.

A comprehensive analysis of LDF grams indicates primary vascular damage of the microhemocirculatory bed, and confirms its role in the occurrence of ulcerative-necrotic changes and hemorrhage of the oral mucosa in patients with hemophilia.

\section{REFERENCES}

1. Babushkin I. E. Medico-psychological status of patients with hemophilia in a comparative aspect: author's thesis of the candidate of medical Sciences. Barnaul, 2001.-238 C.

2. Volokitina N. In. Andreeva T. A. the Provision of dental care to patients with congenital coagulopathies // Probl. gematol. 2004. - N 1. - P. 29-30.

3. Deryabin E. I., Permyakov N. E. Warning lookover of bleeding in patients with hemophilia after tooth extraction // Proc. abstracts Doc. Vseross. nauchno-praktich. konf.: M.: 2003. - P. 40-41.

4. Elizarova V. M., Petrovich Yu. a. Violation of calcium homeostasis in multiple dental caries / / Dentistry. - 2002. N 1. - P. 67-71.

5. Zorenko V. Yu., Reconstructive and restorative treatment of patients with hemophilia; Barnaul, 2007 (Doct.).

6. Kargin V. D., Egorova Jl. B., Nazarova N. S. and others. Pathogenetic features of the course of hemophilia. / / Hematology and Transfusiology. -1997. N 3. - P. 29-32.

7. Klimova E. E., Fedorov D. V., Bishevsky K. N., etc. Changes in bone metabolism in systemic osteoporosis in patients with hemophilia / / Topical issues of radiation diagnostics of diseases of the bone and joint system. Materials of the all-Russian conference. - Barnaul, 2007. - Pp. 76-78.

8. Knyazev C. B., Maksimovsky Yu. M., Volozhin A. I. et al. Experience of using the drug "Colapol" to stop gingival bleeding in patients with hemophilia / / Doctor. - 1993. N 9. - P. 24-25.

9. Lobanova E. V., Chernov V. M., Rumyantsev A. G. Morbidity of children with hemophilia in 17 regions of the Russian Federation in the period from 1991 to 1994. / / Hematology and Transfusiology. 1999. - no. 6. - P. 63.

10. Mazyrko M. A. Comparative aspects of radiation diagnostics of hemophilic arthropathies: dis. .. kand. med. nauk. Barnaul, 2001. - 167 p.

11. Makhmudov A.D., Boboev K. T., Azlarova A. T. Hemophilia: diagnosis, clinic, prevention 
and treatment. Met.recommendations.

Tashkent, 2007, p. 25.

12. Momot A. P., Mamaev A. N., Elykomov V. A., Zaitsev V. I., Belykh V. I., Raspopova E. A., Rozum V. E. Experience of induction of immune tolerance in patients with an inhibitory form of hemophilia a living in the Altai territory / / in the book: Clinical pharmacology and rational pharmacotherapy. Materials of the second Congress of clinical pharmacologists of the Siberian Federal district with international participation. 21-22 may, Barnaul 2009. p. 311-314.

13. Papayan L. P. Modern model of hemostasis and mechanism of action of the drug NovoSeven / / Problems of Hematology and blood transfusion. - 2004. - N 1. - P. 1117. 